Modern Physics Letters A

(C) World Scientific Publishing Company

\title{
BIRTH OF CLOSED STRINGS AND DEATH OF OPEN STRINGS DURING TACHYON CONDENSATION
}

\author{
KOJI HASHIMOTO \\ Institute of Physics, University of Tokyo, Komaba \\ Tokyo, 153-8902, Japan \\ koji@hep1.c.u-tokyo.ac.jp \\ PEI-MING HO AND JOHN E. WANG \\ Department of Physics, National Taiwan University \\ Taipei 106, Taiwan \\ and National Center for Theoretical Sciences, Taiwan \\ National Taiwan University \\ Taipei 10617, Taiwan \\ pmho@phys.ntu.edu.tw, hllywd2@phys.ntu.edu.tw \\ Received (Day Month Year) \\ Revised (Day Month Year)
}

\begin{abstract}
The tremendous progress achieved through the study of black holes and branes suggests that their time dependent generalizations called Spacelike branes (S-branes) may prove similarly useful. An example of an established approach to S-branes is to include a string boundary interaction and we first summarize evidence for the death of open string degrees of freedom for the homogeneous rolling tachyon on a decaying brane. Then, we review how to extract the flat S-brane worldvolumes describing the homogeneous rolling tachyon and how large deformations correspond to creation of lower dimensional strings and branes. These S-brane worldvolumes are governed by S-brane actions which are on equal footing to D-brane actions, since they are derived by imposing conformality on the string worldsheet, as well as by analyzing fluctuations of time dependent tachyon configurations. As further examples we generalize previous solutions of the S-brane actions so as to describe multiple decaying and nucleating closed fundamental strings. Conceptually Sbrane actions are therefore different from D-brane actions and can provide a description of time dependent strings/branes and possibly their interactions.
\end{abstract}

Keywords: Strings, branes, tachyon condensation

PACS Nos.: 11.25.-w, 11.27.+d

\section{Introduction}

Time dependent solutions in string theory have recently been explored. One motivation is that observational cosmology is revealing that potentially most of the energy density in our universe is in the form of dark energy and dark matter which are not well understood. Further evidence confirming these observations would mean that 
to truly be a fundamental theory of gravity and gauge interactions, string theory must address and incorporate dark energy and dark matter.

A related question whose answers will also be of interest in the study of cosmology, is what kind of solvable time dependent backgrounds exist in string theory and what are the phases of matter with which string theory can supply us. One of the major advances along this direction has been the study of D-branes and their decay. Sen has proposed 1 and there has now been further evidence supporting his conjectures, that unstable D-branes in string theory have two important properties. First, a coincident D-brane and anti D-brane, or an unstable D-brane of wrong dimension, both have a worldvolume open string tachyon scalar field which is governed by a tachyon potential. In the case of the bosonic string, the tachyon potential has a local maximum and a local minimum but it is unbounded from below. The mass of the tachyon field is of the order of the string scale. In the case of the superstring, the theory is invariant under $T \rightarrow-T$ and it is believed that the tachyon potential is bounded from below. If the tachyon is homogeneously taken to its minimum value, then the configuration is actually the closed string vacuum with no D-brane and so no apparent physical open string degrees of freedom. Tachyon condensation in string theory is similar to the Higgs mechanism in a field theory in that both remove the tachyon from the spectrum; however, in string theory the infinite number of massive open string states are also simultaneously removed. The second important property is that an inhomogeneous tachyon configuration can represent a lower dimensional BPS brane. For example a kink configuration which interpolates between two vacuum configurations on an unstable Dp-brane represents a BPS D(p-1)-brane, and a tachyon vortex on a brane anti-brane pair is a BPS $\mathrm{D}(\mathrm{p}-2)$-brane.

Having confirmed that it is possible to parametrically change unstable D-branes to the closed string vacuum or lower dimensions D-branes by varying the value of the tachyon field, the next question one would like to answer is what role do dynamics play in regard to these different tachyon configurations and are they connected through physical processes. Starting from a system in a given configuration, what phases can it dynamically evolve between? Not all phases are necessarily connected through dynamical evolution since parametrically varying the tachyon configuration may change the boundary conditions, the total energy and other conserved quantities which are fixed during time evolution.

In almost all interacting theories dynamics are hard to understand and exact solutions are difficult to obtain. Gutperle and Strominger proposed in Ref. 2] however, that string theory (and also field theories) contains a class of interesting Spacelike brane solutions which can be defined in three ways including Dirichelet boundary conditions in time, time dependent tachyon kinks on decaying branes and supergravity solutions showing back reaction of brane decay on spacetime. Shortly thereafter Sen found an exact rolling tachyor $\underline{3}$ boundary conformal field theory describing the homogeneous time evolution of the open string tachyon on an unstable brane. This solution, obtained from a Wick rotation of an array of D-branes, is also called the full S-brane and corresponds to including the time dependent boundary operator 
on the string worldsheet

$$
S=\lambda \int_{\text {boundary }} d \tau \cosh X^{0}
$$

The energy momentum tensor of this solution is

$$
T_{00}=\mathcal{E}, \quad T_{i j}=-p\left(X^{0}\right) \delta_{i j}, \quad T_{i 0}=0
$$

where the function $p\left(X^{0}\right)$ decreases monotonically to zero for large values of $X^{0}$. This solution is unusual as compared to many other time dependent solutions in that the pressure decreases to zero without any oscillations. Interactions on the string worldsheet boundary correspond to turning on the open string tachyon, therefore the rolling tachyon is a homogeneous solution in which the open string tachyon evolves uniformly on the worldvolume of a space filling unstable D-brane. This solution is a bounce since it begins near the tachyon vacuum and climbs part way up the tachyon potential before falling back to the vacuum. One may also interpret the evolution to begin at time $X^{0}=0$. Here the tachyon perturbation is small and so we expect the open string degrees of freedom to be present. Due to the presence of the tachyon potential, as time passes the tachyon value increases and the solution rolls towards the closed string vacuum. This dynamical open string condensation solution therefore represents the homogeneous decay of an unstable brane.

Given the exact solution for the homogeneous decay of the unstable brane, a natural question to further explore is what are the consequences of introducing perturbations and inhomogeneities in the tachyon profile. In the case of a usual spatially varying kink, small perturbations of these spatial kink solutions can be interpreted as waves on or oscillations of a D-brane. On the other hand as we shall review later, larger perturbations of the D-brane can sometimes be interpreted as lower dimensional branes and strings. In comparison, for the case of time dependent kinks even small inhomogeneities can locally drive the tachyon field to different vacua. If the field falls into different vacuum configurations locally this produces a spatial kink (or other codimension) configuration which presumably will have a description as a stable defect. In conventional field theories the inevitability of the production of topological defects is well known and called the Kibble mechanism. A more precise statement is that in a symmetry breaking phase transition the universe will fall into different minima of the vacuum manifold since regions which are sufficiently separated are not in casual contact. For example any topological defects which can exist, such as the boundary domain walls separating the different vacua, will inevitably form. In string theory unstable D-branes are governed by a similar scalar field tachyon, so presumably it is possible that their decay will also produce many lower dimensional defects such as branes and strings. Work along this direction includes Ref. 4, 5] One feature of those solutions however is that they are singular so for example they correspond to tachyon configurations with singularities or caustics.

In the rest of this review we will examine the fate of open string degrees of freedom left over after the tachyon condensation of the rolling tachyon. Then we 
discuss the approach of Refs. [6] and Ref. 7 which is to formulate an action for an Sbrane and search for their classical solutions. In particular these S-branes include the smooth formation of topological defects. Finally we will also provide new solutions generalizing the results of Refs. [6] and 7 .

\section{Death of Open Strings}

The Sen conjectures $\frac{1}{1}$ especially the first property, look self-contradictory at first sight. The very definition of a D-brane is that it is a boundary of the string worldsheet and so should be described using open strings. The disappearance of a D-brane immediately means the disappearance of the open strings, but how do open strings describe their own disappearance? The answer behind this strange question might provide an interesting new description of closed strings - even theories of closed strings without D-branes might be described solely in terms of open strings.

A useful investigative approach to this question is time-dependent tachyon condensation, because this is a smooth way to relate a vacuum with the unstable D-brane at the top of the tachyon potential and the closed string vacuum at the bottom. An interesting result ${ }^{3}$ deduced with the exactly marginal operator (11) is that the pressure of the system at late time is vanishing. This is an indication of the disappearance of the usual physical degrees of freedom of open strings at the end of the tachyon condensation. Sen further proceeded to write an "effective" action whose classical solution reproduces this behavior of the vanishing pressure,

$$
S=\mathcal{T} \int d^{p+1} x V(T) \sqrt{1+\left(\partial_{\mu} T\right)^{2}} .
$$

The potential $V(T)$ is supposed to be of run-away form for large $T, V(T) \sim$ $\exp (-c T)$ with a theory-dependent constant $c$, and $\mathcal{T}$ is the tension of the unstable D-brane. Though this is not a low energy effective action in the usual sense in string theory, ${ }^{\mathrm{a}}$ it has been coupled to gravity and widely applied to cosmological setups due to its interesting kinetic term. Analyzing this action, one can show in particular that there are no plane wave fluctuations around the classical rolling tachyon solution of (3) at late time ${ }^{3}$ which agrees with the expectation that the tachyon minimum acts as the closed string vacuum. This behavior suggests an interesting possibility: the open string tachyon may be described by an effective action with time dependent classical solutions around which physical excitations disappear.

To gain insight into what mechanism comes into play during brane decay in string theory, let us discuss this disappearance of degrees of freedom in the "effective" action in detail. In Ref. 9] it was shown that the fluctuation around the rolling tachyon solution of (3) is governed by the so-called Carrollian contraction of the Lorentz group whose metric is

$$
g_{\mu \nu}=(0,1,1, \cdots, 1) .
$$

${ }^{a}$ See Ref. 8 for discussions. 
The time-time component of a metric denotes the speed of light, thus this system has a vanishing speed of light and fields governed by the metric can no longer propagate; the fluctuations are effectively governed by a Euclidean metric. The Carrollian limit removes the open string excitations in our case here, and this contraction of the light cone may also occur in other systems losing degrees of freedom.

Carrollian behavior means that a tachyon fluctuation of open strings ceases to propagate at the late stage of the rolling tachyon, appearing motionless on the Dbrane when probed by closed strings living in the bulk. This is the death of the open strings suggested by the "effective" tachyon action (3). More precisely, the time-time component of the effective metric approaches zero in a time-dependent manner, so the open strings gradually lose their velocity and finally become immobile.

It is easy to generalize the above statement to also include fluctuations of the gauge field ${ }^{9}$ In string theory, constant gauge field strength can generally be incorporated into any effective action in the form of the open string metric. Thus the action (3) can be generalized to

$$
S=\mathcal{T} \int d^{p+1} x V(T) \sqrt{-\operatorname{det}\left(\eta_{\mu \nu}+F_{\mu \nu}\right)} \sqrt{1+g_{\mathrm{open}}^{\mu \nu} \partial_{\mu} T \partial_{\nu} T},
$$

where the open string metric $g_{\text {open }}^{\mu \nu}$ is given by the field strength

$$
g_{\text {open }}^{\mu \nu} \equiv\left[(\eta+F)^{-1}\right]^{(\mu \nu)},
$$

where the indices $\mu, \nu$ on the right hand side are symmetrized. At late times of the rolling tachyon, the gauge fluctuations here are also governed by the Carrollian metric (44) further limiting the propagating degrees of freedom.

This phenomenon arises in part from an upper limit on the magnitude of $\dot{T}$ in the tachyon actions and contains intriguing physics since standard field theories not coupled to gravity do not have such bounds on the speed of the rolling of scalar fields. ${ }^{\mathrm{b}}$ One direction suggested from this phenomena is to couple it to gravity and see the consequences relevant to cosmology and braneworlds!11 As we stated earlier, however, one might object that the action (3) doesn't stand on a basis firm enough to trust any string theoretical calculation based on it. To properly answer such questions, one should use a tachyon action which is derived from string theory first principles. The tachyon however is not massless but has a string scale mass squared so all field theories for tachyons are not properly valid low energy effective theories. Nevertheless, one can write an off-shell lagrangian for a limited profile of the tachyon field by using a boundary string field theory (BSFT) ${ }^{12}$ For a tachyon linear in a spacetime coordinate, the lagrangian is written as

$$
S=\mathcal{T} \int d^{p+1} x e^{-\frac{T^{2}}{4}} \frac{y 4^{y} \Gamma(y)^{2}}{2 \Gamma(2 y)}, \quad y \equiv\left(\partial_{\mu} T\right)^{2} .
$$

\footnotetext{
${ }^{\mathrm{b}}$ Recently, brane motion in curved gravity background which has a similar speed limit bound 10
} has attracted interest in view of inflationary cosmology. 
This action played a main role in the verification of the second part of the Sen's conjectures, that is, reproduction of D-brane tensions from the tachyon topological defects. 13 Although this action is valid only for the linear tachyon profiles, one might try to do an analysis similar to that performed for (3) by using the time dependent solution found in Ref. 14. Surprisingly, the result of the fluctuation analysis is the same - the fluctuations of the tachyon field and the gauge field are subject to the Carrollian metric (4). Therefore it is expected that the Carrollian contraction is a reasonably accurate description at the late stage of the rolling tachyon resulting in the freezing of some low energy degrees of freedom.

The above analysis however is insufficient since it covers only tachyon and gauge fluctuations, therefore overlooking the possible interactions with other string states. Furthermore, it is not clear if we can really trust the result, because we used the BSFT action (7) or the "effective" action (3) beyond its limit of validity. To overcome these difficulties, one of the authors together with Terashima developed a technique in BSFT to calculate mass-shell conditions of any string excitation on various linear tachyon background profiles! 15 Using this technique, it was shown in Ref. 16 that in fact all the open string excitations are subject to the Carrollian metric in the rolling tachyon background. This then shows that all open string states disappear during tachyon condensation.

The closed string vacuum is a non-perturbative vacuum from the viewpoint of open string theory containing off shell physics, which are naturally explored using schemes such as string field theories. In the case of cubic string field theory 17 the disappearance of the open strings has been described in a different manner, see Ref. 18, In that approach the static vacuum exhibits trivial cohomology describing the disappearance of the physical states around it, while the rolling tachyon solution is not well understood.

\section{Birth of Closed Strings}

In addition to the death of open strings, tachyon condensation is also accompanied by the birth of closed strings. This can be (partially) understood from the viewpoint of unstable D-branes as follows. The rolling tachyon creates electromagnetic fluctuations from the time-varying vacuum, in a way analogous to the creation of cosmological density perturbations during inflation. A scalar field $\phi$ living in an expanding universe has the kinetic term $a^{-2}(t) \dot{\phi}^{2}$ in its Lagrangian density, where $a(t)$ is the scale factor. Due to the factor $a(t)^{-2}$, the canonical quantization is time dependent, and the induced Bogoliubov transformation is responsible for the creation of particles. Similarly, since the conjugate momentum for the gauge potential $A$ is $D \simeq V(T) \dot{A} / \sqrt{1-\dot{T}^{2}}$ for small $E$, which is also time-dependent due to the rolling tachyon, fluctuations of the electric and magnetic fields are created. It is natural to identify closed strings with electric fluxes (and D-strings with magnetic fluxes) on the unstable D-brane upon tachyon condensation. What is needed is a mechanism which confines electric fluxes into infinitesimally thin bundles, which 
can be identified with strings. Assuming this confinement, 19 the closedness of the string is ensured by the conservation of electric flux.

The confinement mechanism can be heuristically understood as a result of electric flux conservation and energy minimization. The Lagrangian density of an unstable D-brane is of the form

$$
\mathcal{L}=-\sqrt{1-E^{2}} \tilde{\mathcal{L}}(T, z), \quad \text { where } \quad z=-\frac{\dot{T}^{2}}{1-E^{2}}, \quad E=\dot{A},
$$

where we assumed spatial homogeneity for simplicity. Without specifying the explicit expression for $\tilde{\mathcal{L}}$, the Hamiltonian and the electric flux are

$$
H=\int \frac{D}{E}, \quad \Phi=\int D, \quad \text { where } \quad D=\frac{\partial \mathcal{L}}{\partial E}=\frac{E}{\sqrt{1-E^{2}}}\left(\tilde{\mathcal{L}}-2 z \frac{\partial \tilde{\mathcal{L}}}{\partial z}\right) .
$$

Apparently, in order to minimize the energy for given flux $\Phi$, one needs $|E|$ to be close to its maximal value 1 . However, $|E| \rightarrow 1$ implies that $|D| \rightarrow \infty$. For given $\Phi$, this means that the flux is localized in a thin strip of infinitesimal width.

It is remarkable that the unstable D-brane worldvolume theory can describe the process of its decaying into closed strings, but it is even more remarkable that, as we will see, this process is captured by a class of solutions of the S-brane action, which only describes the $T=0$ subspace on the unstable D-brane worldvolume.

The analysis in the previous section of the death of open strings holds in the presence of approximately uniform brane decay. Previously we mentioned the possible role inhomogeneities in defect formation during tachyon condensation. Our approach is to include inhomogeneities in the tachyon decay process and find a controlled way to describe their effects on the rolling tachyon background.

Let us begin with some qualitative remarks on defect formation for a simple model, a scalar field $\Phi$ governed by a potential with a local maximum between two local minima. In a local neighborhood $\Phi$ is perched near the maximum and then time evolves into the two local minima forming a defect. Important features of the process can be understood just by following the regions, $\mathcal{S}$, where the scalar field is at the local maximum. To understand this point it helps to first consider the case where $\Phi$ is sufficiently homogeneous so no defects are generated in the local neighborhood. $\mathcal{S}$ in this case is a spacelike worldvolume giving information only about when the field $\Phi$ was at the maximum. Now introduce small inhomogeneities which lead to the creation of defects. For $\mathcal{S}$ these perturbations are initially small, changing the spacelike trajectory only slightly. As the perturbation grows larger and the defect is created, however, $\mathcal{S}$ is deformed to a timelike worldvolume along the trajectory of the defect. This is a partial deformation of $\mathcal{S}$ in the time direction of the otherwise spacelike worldvolume. While the spacelike regions of $\mathcal{S}$ do not describe any defects, the timelike regions can be interpreted as solitonic defects. Therefore a general feature of the time dependent symmetry breaking process and the formation of defects, is that existence of worldvolumes $\mathcal{S}$ with regions of both spacelike and timelike signatures. 


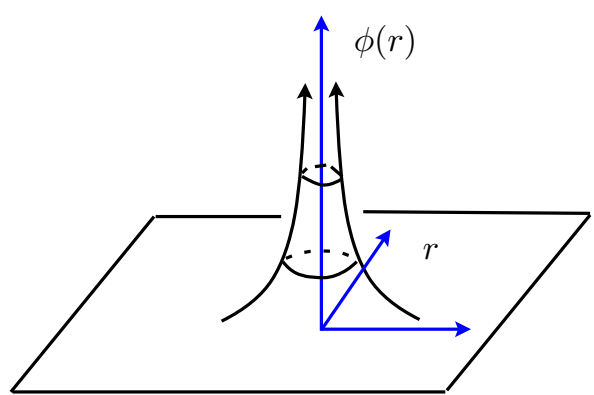

Fig. 1. Intersection of a D-brane and fundamental string.

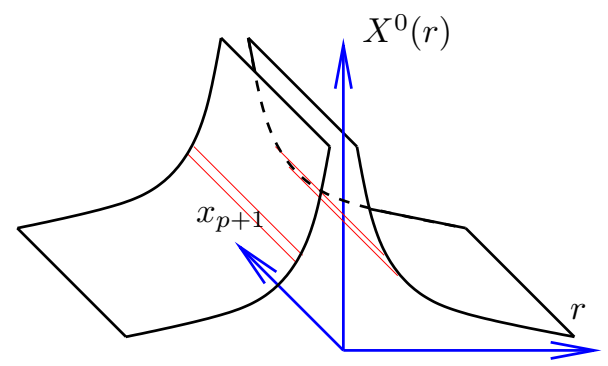

Fig. 2. Intersection of an S-brane and fundamental string. This shows the formation of a fundamental string during timedependent tachyon condensation.

Our viewpoint will be to analyze in string theory the region $\mathcal{S}$ where the open string tachyon is at the maximum of its potential, $T=0$. In the case of a static kink configuration of the tachyon theory, such a region is simply the location of the (lower dimensional) BPS D-brane. BPS D-branes obey the well-known DBI (DiracBorn-Infeld) action in the limit where backreaction can be ignored. This comes from the fact that to preserve conformal invariance of the string action in the presence of boundaries, the boundaries are subject to equations of motion which can be derived from the DBI action. In the so called Monge (or static) gauge, the DBI action can be written with the excitation of a scalar field associated to fluctuations in directions transverse to a flat D-brane worldvolume hypersurface

$$
S=\mathcal{T}_{\mathrm{D} p} \int d^{p+1} x \sqrt{-\operatorname{det}\left(\eta_{\mu \nu}+\partial_{\mu} \phi \partial_{\nu} \phi+F_{\mu \nu}\right)}=\mathcal{T}_{\mathrm{D} p} \int d^{p+1} x \sqrt{-\operatorname{det}(g+F)} .
$$

The Dp-brane is parametrized by $p+1$ worldvolume coordinates $x_{i}(i=0,1, \cdots, p)$, $\mathcal{T}_{\mathrm{D} p}$ is the brane tension, the gauge field strength is $F_{\mu \nu}$ and the fluctuation of the brane in a transverse spatial direction is specified by the scalar field $\phi$. Due to the curving of the D-brane worldvolume along the $\phi$ direction, the induced metric on the brane is $g_{\mu \nu}=\eta_{\mu \nu}+\partial_{\mu} \phi \partial_{\nu} \phi$. In the limit of a flat D-brane with no excitations, $\phi=0$, this clearly gives a well defined action for time-like extended D-branes.

Solutions to the DBI action include the very interesting BIon 2021

$$
\phi=A_{0}=\frac{b_{p}}{r^{p-2}}
$$

which is a perpendicular intersection of a D-brane and a fundamental string. See Fig. 1 Here $A_{0}$ is the Coulomb potential, $b_{p}$ is the electric charge, and $r$ is the radial coordinate $r \equiv \sqrt{x_{1}^{2}+\ldots+x_{p}^{2}}$. This solution is an example of how open string degrees of freedom on the D-brane can describe a closed string.

Primarily only timelike D-branes have been examined, but with the introduction of S-branes, the relevance of also constructing classical solutions of the equation of motion which have spacelike worldvolumes has become more clear. It is possible 
to show that spacelike solutions to the equations of motion exist and in fact are classical solutions of the Euclidean DBI action

$$
S=S_{0} \int d^{p+1} x \sqrt{\operatorname{det}\left(\delta_{\mu \nu}-\partial_{\mu} X^{0} \partial_{\nu} X^{0}+F_{\mu \nu}\right)}=S_{0} \int d^{p+1} x \sqrt{\operatorname{det}(g+F)}
$$

where $S_{0}$ is a normalization constant. The worldvolume is parametrized by the coordinates $x^{i}, i=1, \ldots, p+1$ and so now transverse excitations can be along the timelike direction $X^{0}$. In the limit of no fluctuations, $X^{0}=0$, the worldvolume is spacelike so these are Spacelike-branes.

While Euclidean solutions stand on a firm basis and arise very naturally, they typically are considered to be associated to quantum effects such as instantons. ${ }^{c}$ As a result of the introduction of the notion of S-branes, however, it is now possible to interpret some of these Euclidean solutions as classical solutions in ordinary spacetime instead of as instantons. The important difference is to look at Euclidean solutions embedded in background spacetimes which are timelike and with timelike excitations. In fact it has been shown that the Euclidean DBI action naturally arises during time dependent tachyon condensation and has been derived from the boundary string field theory and related tachyon actions as discussed in Ref. [6. To understand how Euclidean solutions are related to classical solutions, let us recall the homogeneous time dependent tachyon kinks. Although naively a flat S-brane is an object which is moving with infinite velocity, by examining its associated rolling tachyon there is in fact no violation of causality or even energy transfer associated with this solution. This Euclidean hypersurface merely corresponds to the homogeneous time evolution of the tachyon from its $T=0$ unstable maximum (which one may call a "time dependent tachyon kink").

If the gauge field is turned off, then spacelike solutions of the S-brane equation of motions in Lorentzian backgrounds are called maximal surfaces in the literature since variations of the embedding generically reduce the worldvolume. We will be interested in solutions which change their worldvolume signature with respect to the background geometry and so these will also be timelike solutions to the S-brane equations of motion and have been less studied.

Solutions which change signature are allowed in the absence of gauge fields and the equations of motion are satisfied across the transition point ${ }^{22}$ These solutions however go through a zero in the action around which higher order effects could be important. In contrast, by turning on a gauge field on the S-brane, it is possible to obtain solutions to the equations of motion which smoothly change signature and always have finite action. On the worldvolume of a D-brane, gauge fields can be interpreted as lower dimensional D-branes and strings. Gauge fields on an S-brane will have a similar interpretation as being branes and strings. We next discuss Sbrane solutions which exist for long periods of time and describe time dependent

${ }^{c}$ The euclideanized DBI has been used for various instanton effects, especially in compactification of target space, as D-branes wrapping various cycles in Calabi-Yau manifolds. These are relevant to what is called worldsheet instantons. 
defect formation associated to the presence of gauge fields.

Intersecting S-branes solutions analogous to the BIon solutions were found in Ref. [6. For the cases $p \geq 3$ the solution is

$$
X^{0}=A_{p+1}=\frac{c_{p}}{r^{p-2}}
$$

where $A_{p+1}$ is the electric gauge field along the $p+1$-direction whose strength is parameterized by $c_{p}$. Here $r=\sqrt{\left(x_{1}\right)^{2}+\cdots+\left(x_{p}\right)^{2}}$ as before, but note that $x_{p+1}$ is not included in the definition. See Fig. 2 The induced metric on the S-brane is

$$
d s^{2}=d x_{p+1}^{2}+\left(1-\frac{c_{p}^{2}}{r^{2 p-2}}\right) d r^{2}+r^{2} d \Omega_{p-1}^{2}
$$

which is spacelike for $r>\left(c_{p}\right)^{1 /(p-1)}$ (or equivalently $0<X^{0}<\left(c_{p}\right)^{1 /(p-1)}$ ) and timelike for smaller radial values (later time). This metric clearly describes a cylindrical tube collapsing into a thin line. When the radius is small, this corresponds to being at late time and far from the original appearance of the S-brane surface. Although the metric changes signature we stress that the action is finite and smooth across the signature change. The induced electric field strength of the solution, $F_{X^{0} x^{p+1}}=1$ in the unit $2 \pi \alpha^{\prime}=1$, is the critical electric field value. In fact the tension generated by this electric field is exactly the fundamental string tension! As in the case of the BIon, this energy condition is a result of charge quantization along the S-brane worldvolume. This S-brane therefore also has an interpretation as an example of the confinement of electric flux into a fundamental string. While previous BIon solutions were intersections of D-branes and fundamental strings, these new solutions can properly be called intersections of S-branes with fundamental strings.

From our discussion of the Carrollian limit, we can provide an intuitive explanation for the existence of solutions which smoothly change worldvolume signature. As defined in the context of the worldvolume action, the spacelike brane is a solution which is spacelike relative to the open string metric, $g_{\mu \nu}^{\text {open }}$ and not the background closed string metric $\eta_{\mu \nu}$. In the absence of a gauge field, both metrics are equal so a spacelike brane can be spacelike relative to the closed string metric. If the gauge fields are turned on however, the open and closed string metrics are not equal. This then allows for the existence of an classical S-brane which is spacelike relative to the open string metric and yet timelike relative to the closed string metric.

More precisely, the light-cone defined by the open string metric lies inside the light-cone of the closed string metric; 23 the velocity of light on the brane is smaller than that in the bulk by the ratio $\sqrt{1-E^{2}}$. (When $E^{2}=1$, like in the Carrollian limit, open string degrees of freedom stop propagating along the directions transverse to the electric field.) It is therefore possible for a superluminal fluctuation on the brane to obey the causality constraints in the bulk. For such configurations, the S-brane Lagrangian is real (and the usual D-brane Lagrangian is imaginary).

When the tachyon condenses homogeneously, the usual open string degrees of freedom die due to the closing of the open string light cone in the Carrollian limit. 
In contrast, the closing of the light cone effects these S-brane solutions differently, so it again appears that closed strings have a description in terms of open string degrees of freedom on the brane. Therefore while open string degrees of freedom may die, under small perturbations closed string degrees of freedom may be born during the tachyon condensation process!

\section{New solutions}

There are two well known extensions of the D-brane BIon solutions given above. BIons are supersymmetric as is typical for harmonic function type solutions. It is therefore possible to construct an arbitrary configuration of fundamental strings all in parallel to one another and perpendicularly intersecting the D-brane worldvolume. Due to the fact that the solutions are supersymmetric, an arbitrary configuration of strings can be represented by simply summing the above harmonic solutions together for any number of individual strings.

Surprisingly even though these intersecting S-brane configuration are not supersymmetric, it is again possible to construct a multiple spike solution by adding the above harmonic functions together to obtain

$$
X^{0}=A_{p+1}=\sum_{a} \frac{c_{p}^{a}}{\left[\left(x_{1}-x_{1}^{a}\right)^{2}+\ldots .+\left(x_{p}-x_{p}^{a}\right)^{2}\right]^{(p-2) / 2}} .
$$

The strings are labeled by the integer $a$. This solution has an interesting spacetime interpretation. It begins as an approximately cylindrical tube similar to the formation of a single string. As time evolves however local bumps and structures develop on the tube until eventually the S-brane separates into many disconnected tubes (see Fig. 31). From a spatial point of view this would appear to be a change in topology proceeding in the sequence $R \times S^{p-1} \rightarrow R \times\left(S^{p-1} \oplus S^{p-1}\right) \rightarrow \cdots \rightarrow R \times \Sigma_{i}\left(S^{p-1}\right)_{i}$. Although the cylinders become spatially disconnected, from the spacetime point of view they are all described by a single smooth S-brane embedding.

When some of the parameters $c_{p}^{a}$ become negative, the corresponding spikes in the time direction are oriented towards the past. This is the situation where we have

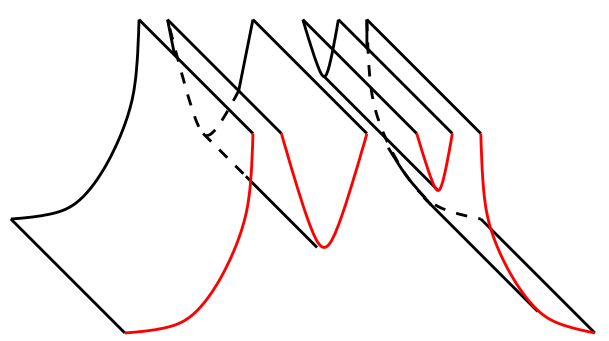

Fig. 3. Several closed strings created during the rolling tachyon process. While the S-brane worldvolume looks disconnected in the figure it is actually connected; this figure does not show the angular directions.

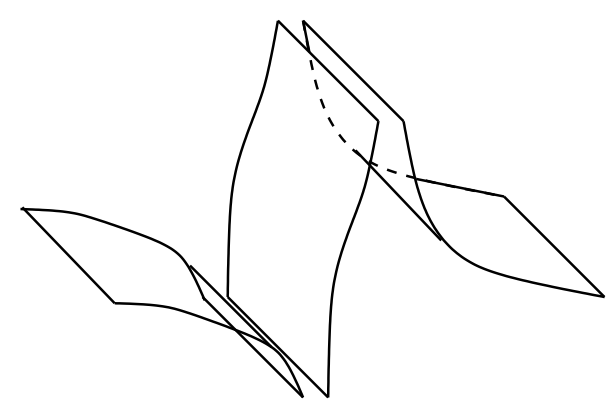

Fig. 4. A closed string also present in the past with $c_{p}<0$. 
several closed strings also in the past and they decay once before finally forming other closed strings in the future (see Fig. (4).

In addition to the above BIons, it is possible to find solutions which are not infinitely extended in space or time. By decreasing the ratio of charge to mass in (11), the solution becomes a D-brane and anti-D-brane pair connected by a throat. 20 Although the solution is not supersymmetric it is static; similar solutions representing the annihilation of D-branes and anti-D-branes have also been found 24 Our intersecting S-brane has a similar deformation which is given by

$$
X^{0}=\int \frac{b_{p} d r}{\sqrt{r^{2 p-2}-r_{0}^{2 p-2}}}, \quad A_{p+1}=\int \frac{a_{p} d r}{\sqrt{r^{2 p-2}-r_{0}^{2 p-2}}}
$$

where $r_{0}^{2 p-2}=a_{p}^{2}-b_{p}^{2}$. See Fig. 5 This S-brane has an interpretation as a partial formation of a string. There is a local fluctuation away from the vacuum which tries but does not succeed in properly interpolating between different vacua. All of these solutions undergo worldvolume signature change from spacelike to timelike worldvolume for $r_{0}<r<b_{p}^{1 /(p-1)}$. The local fluctuation only exists for a finite amount of time before disappearing.

While these S-brane solutions can be smooth, the solutions of Refs. 4, 5] describing the creation of co-dimension one branes contain singularities. Let us examine under what conditions the D-brane and S-brane worldvolumes are smooth and when they contain caustics and singularities. The D-brane and anti-D-brane pair for example satisfy a constraint $r_{0}^{2 p-2}=b_{p}^{2}-a_{p}^{2}$ relating the radius of the throat $r_{0}$, the height of the throat which is determined by $b_{p}$ and the electric flux $a_{p}$. For the parameter range $b_{p}>a_{p}$, which can be thought of as a subextremal limit, the throat is smooth. The superextremal solutions $b_{p}<a_{p}$ describe one D-brane which comes together forming a caustic ${ }^{2025}$ past which we do not properly understand what should happen (Fig. [6). On the other hand S-branes obey the constraint $r_{0}^{2 p-2}=a_{p}^{2}-b_{p}^{2}$ so smooth solutions, $a_{p}>b_{p}$, are those where the field strength is superextremal and larger than the timelike fluctuation. Singular S-brane configurations are subextremal with $a_{p}<b_{p}$ (see Fig. 7). Given the existence of smooth S-brane solutions, however, one hopes and expects that related non-singular solu-

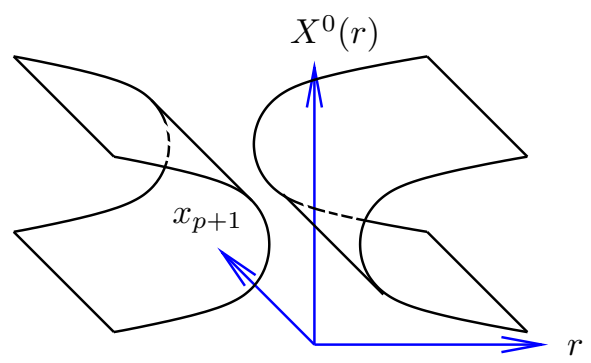

Fig. 5. A partial formation of a string while it decays away. 
tions will be found using approaches similar to that in Ref. 4, 5.

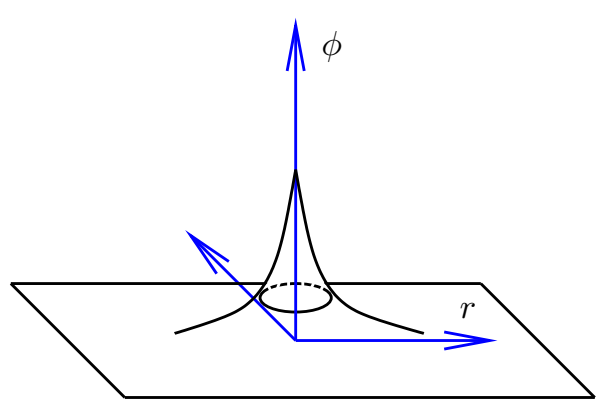

Fig. 6. A static BIon with a cusp at the top.

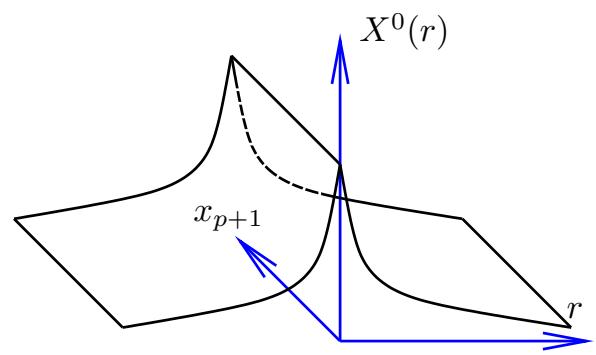

Fig. 7. A caustic is formed at a finite time, in a solution of the S-brane action.

\section{Discussions}

By interpreting the role of Euclidean D-brane solutions during brane decay and also explicit derivation from tachyon actions, we have shown that the S-brane actions, first used and applied in Refs. [6] and [7, allow for solutions describing the emergence of some closed string physics. In view of the fact that D-brane actions are fruitfully applied for many results in string theory, we are potentially still only scratching the surface, and it would be interesting to see what further role S-brane actions have in physics. The equations of motion coming from the S-brane action are the same as those from the D-brane action, thus at some level they are basically the same. Conceptually, however, the S-brane actions are different and so they potentially open up new directions for time dependent D-brane dynamics. Due to its worldvolume parametrization, for example, S-branes can simultaneously describe spatially disconnected objects. We also emphasize that S-branes are not instantons due to the background metric and fluctuations in the time direction. This makes it possible to describe with ease the formation/annihilation of D-branes/strings as seen in this review; it would be interesting to consider possible connections to string production during inflation. ${ }^{26}$ Since D-branes helped give rise to many developments in string theory, such as AdS/CFT, holography, Matrix theory, braneworlds and many other topics, S-branes potentially may bring more than just generalizations of the previous topics. S-brane actions stand on the same footing as the D-brane actions, and so we expect the possibility for their use in many as yet undiscovered applications.

The $\beta$-function approach of string sigma models gives the target space equations of motion, and in this sense the S-brane actions are qualified to work at the same level as the usual D-brane actions. But, as long as the S-brane action is intimately related to the D-brane decay, it is important to clarify the relation to the picture of time dependent tachyon condensation and its deformation. The derivation of the action given in Ref. [6 was based on a homogeneous rolling tachyon, while the S-brane solutions considered in this review have large deformations from the homogeneous 
configuration. Thus it is not straightforward to construct corresponding tachyon solutions, as opposed to the situation of the static BIons. 27 A subtle problem here is that there is no "tachyon effective theory" since the tachyon already has a mass squared of order string scale. This suggests that one has to use string field theories to describe inhomogeneous tachyon condensation which in general appears quite difficult. Our S-brane approach, we believe, may extract an important part of the dynamics in a consistent manner with the string field theories. We also note that the S-brane approach may be applied to various field theories with topological defects, not only string theory, because the derivation given in Ref. [6 is quite general. Applications along this direction should be intriguing.

Although we have discussed the open string theory degrees of freedom during tachyon condensation, there is no known decoupling limit of closed string modes in this case. In fact it is an important and unresolved question as to what exactly is the endstate of tachyon condensation called tachyon matter. In Ref. 28 it was argued that this matter is unstable and that the energy in the tachyon condensation process will be transferred into very massive closed strings which then disperse into the bulk. Alternately Sen argues that while the energy is transferred into closed strings, their dispersal is slow and in fact tachyon matter is a specific form of stable closed strings which can be described by open strings. From this viewpoint there may be a possible new open closed string duality where a given open string background has a closed string dual. ${ }^{29}$ An example of where this proposal has been checked is in the $c=1$ matrix models. Also, in trying to study effects of gravitational backreaction on the brane decay process, candidate dual supergravity solutions have also been constructed in Ref. 30] where it was found that their decay time could be infinitely extended through explicit scaling limits; insight into the possibility of a new open closed string duality could arise from a careful study of their near horizon limit. Recently Ref. 31 has also argued that electric fields act as a catalyst for the decay of unstable D1-branes decay into closed strings and further increase the stability of the tachyon condensate. In general though the endpoint of tachyon condensation is still a problem needing investigation.

With the inclusion of gauge fields on the D-brane worldvolume, even if tachyon matter is unstable, however, the production of closed strings is still at the heart of these S-brane solutions.

\section{Acknowledgments}

K.H. would like to thank his collaborators concerning this subject, G. W. Gibbons, S. Nagaoka, S. Terashima, and P. Yi. The work by K.H. was supported in part by the Grant-in-Aid for Scientific Research (No. 12440060, 13135205, 15540256 and 15740143) from the Japan Ministry of Education, Science and Culture. P.M.H. and J.E.W are supported in part by the National Science Council, the Center for Theoretical Physics at National Taiwan University and the National Center for Theoretical Sciences. 


\section{References}

1. A. Sen, JHEP 9808, 012 (1998); Int.J.Mod.Phys. A14, 4061 (1999); hep-th/9904207 JHEP 9912, 027 (1999).

2. M. Gutperle and A. Strominger, JHEP 0204, 018 (2002).

3. A. Sen, JHEP 0204, 048 (2002); JHEP 0207, 065 (2002); MPL A17, 1797 (2002).

4. A. Sen, JHEP 0210003 (2002),

P. Mukhopadhyay and A. Sen, JHEP 0211047 (2002),

S.-J. Rey and S. Sugimoto, Phys. Rev. D68 026003 (2003).

5. F. Larsen, A. Naqvi and S. Terashima, JHEP 0302039 (2003).

6. K. Hashimoto, P. -M. Ho and J. E. Wang, PRL 90141601 (2003).

7. K. Hashimoto, P. -M. Ho, S. Nagaoka and J. E. Wang, Phys. Rev. D68 026007 (2003).

8. D. Kutasov and V. Niarchos, Nucl. Phys. B666 56 (2003).

9. G. W. Gibbons, K. Hashimoto and P. Yi, JHEP 0209, 061 (2002).

10. E. Silverstein and D. Tong, hep-th/0310221

M. Alishahiha, E. Silverstein and D. Tong, hep-th/0404084

11. G. W. Gibbons, Phys.Lett. B537, 1 (2002); Class.Quant.Grav. 20, S321 (2003).

12. E. Witten, Phys.Rev. D46, 5467 (1992); Phys.Rev. D47, 3405 (1993);

S. L. Shatashvili, Phys.Lett. B311 83 (1993); hep-th/9311177

13. D. Kutasov, M. Marino and G. Moore, JHEP 0010, 045 (2000) hep-th/0010108

A. A. Gerasimov and S. L. Shatashvili JHEP 0010, 034 (2000);

P. Kraus and F. Larsen, Phys.Rev. D63, 106004 (2001);

T. Takayanagi, S. Terashima and T. Uesugi, JHEP 0103, 019 (2001).

14. S. Sugimoto and S. Terashima, JHEP 0207, 025 (2002);

J. A. Minahan, JHEP 0207, 030 (2002).

15. K. Hashimoto and S. Terashima, hep-th/0408094

16. K. Hashimoto and S. Terashima, JHEP 0406, 048 (2004).

17. E. Witten, Nucl.Phys. B268, 253 (1986).

18. H. Hata and S. Teraguchi, JHEP 0105, 045 (2001);

I. Kishimoto and T. Takahashi, Prog. Theor.Phys. 108, 591 (2002).

19. P. Yi, Nucl. Phys. B 550, 214 (1999);

O. Bergman, K. Hori and P. Yi, Nucl. Phys. B 580, 289 (2000);

G. W. Gibbons, K. Hori and P. Yi, Nucl. Phys. B 596, 136 (2001);

A. Sen, J. Math. Phys. 42, 2844 (2001).

20. C. G. Callan and J. Maldacena, Nucl.Phys. B513, 198 (1998).

21. G. W. Gibbons, Nucl.Phys. B514, 603 (1998).

22. G. Gibbons and A. Ishibashi, Class. Quant. Grav 212919 (2004).

23. G. W. Gibbons and C. A. R. Herdeiro, Phys.Rev. D63 064006 (2001).

24. K. G. Savvidy, hep-th/9810163

K. Hashimoto, JHEP 0207, 035 (2002);

J. E. Wang, JHEP 0210, 037 (2002).

25. A. Hashimoto, Phys.Rev. D57, 6441 (1998).

26. S. Sarangi and H. Tye Phys. Lett. B536, 185 (2002);

E. J. Copeland, R. C. Myers and J. Polchinski JHEP 0406, 013 (2004).

27. K. Hashimoto and S. Hirano, JHEP 0004003 (2001), Phys.Rev. D65 026006 (2002).

A. Sen, Phys.Rev. D68 106003 (2003).

28. N. Lambert, H. Liu and J. Maldacena, hep-th/0303139

29. A. Sen, Phys.Rev.Lett. 91, 181601 (2003).

30. G. Jones, A. Maloney and A. Strominger, Phys. Rev D69 126008 (2004);

G. Jones and J. E. Wang, hep-th/0409070

31. M. Gutperle and P. Yi, hep-th/0409050 